This is an electronic reprint of the original article. This reprint may differ from the original in pagination and typographic detail.

Author(s): Puttaraksa, Nitipon; Whitlow, Harry J.; Napari, Mari; Meriläinen, Leena; Gilbert, Leona

Title: Development of a microfluidic design for an automatic lab-on-chip operation

Year: $\quad 2016$

Version:

Please cite the original version:

Puttaraksa, N., Whitlow, H. J., Napari, M., Meriläinen, L., \& Gilbert, L. (2016).

Development of a microfluidic design for an automatic lab-on-chip operation.

Microfluidics and Nanofluidics, 20(142). https://doi.org/10.1007/s10404-016-1808-0

All material supplied via JYX is protected by copyright and other intellectual property rights, and duplication or sale of all or part of any of the repository collections is not permitted, except that material may be duplicated by you for your research use or educational purposes in electronic or print form. You must obtain permission for any other use. Electronic or print copies may not be offered, whether for sale or otherwise to anyone who is not an authorised user. 


\title{
Development of a microfluidic design for an automatic lab-on-chip operation
}

\author{
Nitipon Puttaraksa • Harry J. Whitlow • \\ Mari Napari • Leena Meriläinen • Leona \\ Gilbert
}

Received: 1 June 2016 / Accepted: 22 September 2016

\begin{abstract}
Simple and easy to use are the keys for developing lab-on-chip technology. Here, a new microfluidic circuit has been designed for an automatic lab-onchip operation (ALOCO) device. This chip used capillary forces for controlled and precise manipulation of liquids, which were loaded in sequence from different flowing directions towards the analysis area. Using the ALOCO design, a non-expert user is able to operate the chip by pipetting liquids into suitable inlet reservoirs. To test this design, microfluidic devices were fabricated using the programmable proximity aperture lithography (PPAL) technique. The operation of the ALOCO chip was characterized from the flow of red-, blue- and un-dyed deionized (DI) water. Experimental result indicated that red water, which filled first the analysis area, was substituted entirely with blue water. Controlled sequential flows of these water in the ALOCO device are demonstrated in this paper.
\end{abstract}

Keywords Microfluidics · Capillary flow · Lab-on-chip · MeV ion beam lithography

N. Puttaraksa (Corresponding author)

Department of Biological and Environmental Science and Nanoscience Center, University of Jyvaskyla, Jyvaskyla, Finland

E-mail: nitipon.n.puttaraksa@jyu.fi

H.J. Whitlow

Department of Physics, University of Jyvaskyla, Jyvaskyla, Finland

Present address: of H.J. Whitlow Louisiana Accelerator Center, University of Louisiana at Lafayette, P.O. Box 43600, Lafayette, Louisiana 70504-43600, USA

M. Napari

Department of Physics, University of Jyvaskyla, Jyvaskyla, Finland

L. Meriläinen, and L. Gilbert

Department of Biological and Environmental Science and Nanoscience Center, University of Jyvaskyla, Jyvaskyla, Finland

Present address: of L. Meriläinen Institute of Biotechnology, University of Helsinki, 00014 Helsinki, Finland 


\section{Introduction}

The scaling down of biological and chemical experiments into small devices, namely lab-on-chip (LOC), has many advantages over conventional laboratory experiments (Dittrich and Manz 2006; Chin et al. 2007; Jiang et al. 2011). The miniaturization implies that the amount of costly reagents can be reduced to the order of $\mu \mathrm{L}$ or less (Stone et al. 2004). Working with such tiny volumes can give a dramatic decrease in cost and increase in the speed of analysis (Sackmann et al. 2014). The use of LOC platforms has found applications in many fields, e.g. drug development (Weigl et al. 2003), proteomics (Mouradian 2001), and clinical diagnostics (Srinivasan et al. 2004). The LOC technology generally uses microfluidics for manipulating liquids flowing in microchannels (Squires and Quake 2005; Whitesides 2006). Actuation and control of the flow of liquids in such devices can be hydraulic (syringe pump) (Puttaraksa et al. 2012), electrokinetic (Bousse et al. 2000), acoustic (Yeo and Friend 2009), centrifugal (Cho et al. 2007), and capillary (Juncker et al. 2002; Puttaraksa et al. 2013). However, many of these methods require external components, e.g. syringe pump for hydraulic flow, rotator to create centrifugal force, and electrical supply for electrokinetic flow. The use of external actuation components has significant drawbacks such as additional external connections to the chip, which reduces reliability, increases electrical power consumption, as well as decreases compactness and portability (Jin et al. 2012; Novo et al. 2013). Capillary force actuation of liquids is particularly interesting because liquids can be transported spontaneously in microchannels without the need for any external components. The flow can be regulated in a controlled manner through the surface tension of the liquids, wettability of the walls of the microchannels, as well as channel height and width (Delamarche et al. 1998). Capillary microfluidic chips have the potential to integrate a wide range of microfluidic components, in a single chip (e.g. two to four-way junctions, analysis chambers, liquid inlet- and outletreservoirs, pumps, as well as valves). The combination of lithographically defined capillary microfluidics, where the flow volumes are precisely controlled by the geometry of the capillary microfluidic components, makes an intrinsic volumetric analysis capability.

A microfluidic device using capillary system for autonomous sequential delivery of different liquids into a reaction chamber has been introduced by Juncker et al. (2002). Then, a similar concept has been presented for immunoassay application in one step by Zimmermann et al. (2009). These devices required a treatment of ethanolic solution of thiolated poly(ethylene glycol) for producing hydrophilicity of Au surface in the capillary system (Juncker et al. 2002; Zimmermann et al. 2009). A capillary flow microfluidic chip used together with an absorbent pad was fabricated in poly(methyl methacrylate) or PMMA for detection of nucleic acid, as reported by Jin et al. (2012). This device also modified the PMMA surface by treatment with UV/ozone for increasing the hydrophilicity (Jin et al. 2012). Lately, Novo et al. (2013) have demonstrated a capillary microfluidic chip that enabled autonomous and sequential flowing liquids. However, the device was made of polydimethylsiloxane (PDMS), which is hydrophobic. In order to flow liquids in this material by the capillary forces, the PDMS surface requires modification i.e. by UV-ozone treatment (Novo et al. 2013). These surface modification processes could increase the additional steps for the production of microfluidic chips and consequently alter the surface for biological competences. 
To eliminate the surface treatment/modification, microfluidic devices made of Si and PMMA materials have been developed. The devices were fabricated for testing using $\mathrm{MeV}$ ion beam lithography with the programmable proximity aperture lithography (PPAL) technique (Puttaraksa et al. 2008). Open microchannel patterns were firstly made in PMMA resist coated on $\mathrm{Si}$ wafer (Whitlow et al. 2009). Then, sealed channel PMMA/PMMA/Si microfluidic chips were produced by capping the open microchannel pattern with thermally bonded PMMA film. Microfluidic chips made by this approach allow spontaneous liquid filling of the microchannels without any extra surface treatments or modifications (Puttaraksa el al. 2013). Moreover, mass production of the channel structures is possible by so-called nanoimprint lithography (NIL) (Ansari et al. 2006) or aperture/stencil mask $\mathrm{MeV}$ ion beam lithography (Brun et al. 2013). Additionally, fabrication of PMMA/PMMA/Si microfluidic device using the PPAL technique is a fast development of a microfluidic prototype because of its maskless lithography method (Puttaraksa et al. 2013). The microfluidic prototype could be used for high-throughput fabrication in PMMA e.g. by using hot embossing (Becker and Heim 2008). For this reason, the production of capillary microfluidic device in PMMA/PMMA/Si by the PPAL approach could consider being more advantages than the standard fabrication of PDMS microfluidic device using soft lithography. This is because not only the UV-ozone treatment is required to modify the surface of PDMS for hydrophilicity but also the soft lithography is not suitable for high-throughput fabrication. Furthermore, mass productions, e.g. hot embossing and injection molding are not applicable for fabrication of PDMS microfluidic chips (Sackmann et al. 2014).

The goal of this paper is to present a new microfluidic chip that exploits PMMA/PMMA/Si capillary-force actuated microfluidics for automatic lab-onchip operation (ALOCO). With this design, it is demonstrated that by loading liquids into different inlet reservoirs in sequence, the liquids are automatically manipulated to successively flow into a set of analysis volumes. This provides a simple and easy-to-use device without any external actuation devices that is suitable for non-expert users. The ALOCO chip concept is suitable for standard analytical applications such as immunoassay, sequential and batch assays for food quality, as well as environmental monitoring.

\section{Experimental}

Microfluidic patterns were fabricated in a PMMA layer coated on $\sim 1 \mathrm{~cm} \times \sim 1$ $\mathrm{cm}$ silicon wafer using the PPAL method (Puttaraksa et al. 2008). The PMMA films of $\sim 9 \mu \mathrm{m}$ thickness were produced as previously described (Puttaraksa et al. 2013). The samples were irradiated with an ion beam of $3 \mathrm{MeV}^{4} \mathrm{He}^{2+}$ ions at an ion fluence of $\sim 2.5 \times 10^{13}$ ions $/ \mathrm{cm}^{2}$ for making latent image of desired structures. Then, a 7:3 isopropyl alcohol/deionised (DI) water mixture was used to dissolve the PMMA in the irradiated area, resulting in open channels of microfluidic circuits. Subsequently, the chips were rinsed in DI water and dried with a flow of helium gas. The open microchannel structures were then capped with a PMMA film of $50 \mu \mathrm{m}$ thickness from Goodfellow ${ }^{T M}$, to form closed channels. This process was completed by using thermal bonding on a hotplate at $\sim 110{ }^{0} \mathrm{C}$ (Puttaraksa et al. 2013). To follow the flow of liquids in the chips, red-, blue- and un-dyed DI 
water was used. A microscopic system, composing of a digital video camera (Canon Powershot A520) connected with a Brunle SP-400 optical microscope, was used to record images and movies, which were consequently analyzed using the open source ImageJ software (NIH) (Abramoff et al. 2004).

Here, liquids were propelled through the microfluidic circuits by capillary forces. Principally, capillary flow dynamics relates to the capillary pressure $\left(p_{c}\right)$, which is defined by the properties of the channels (i.e. geometries and contact angles) as well as liquid surface tension (Delamarche et al. 1998). The capillary pressure as reported by Delamarche et al. (1998) can be applied to the microfluidic chips used in this work as:

$$
p_{c}=\gamma\left(\frac{\cos \theta_{S i}+\cos \theta_{P M M A, \text { Goodfellow }}}{h}+\frac{2 \cos \theta_{P M M A}}{w}\right),
$$

where $\gamma$ is the surface tension, $\cos \theta_{S i}$ is the contact angle of $\mathrm{Si}, \theta_{P M M A}$, Goodfellow is the contact angle of PMMA film from Goodfellow, $\theta_{P M M A}$ is the contact angle of PMMA, as well as $h$ and $w$ are the height and width of the channel, respectively. The positive capillary pressure $\left(p_{c}>0\right)$ is a driving force to move the liquid meniscus in the microchannel, whereas the negative capillary pressure $\left(p_{c}<0\right)$ is a retarded force to prevent the moving of the liquid meniscus in the microchannel (Feng et al. 2003). Capillary valves (CV) were used to control the liquid flows in the microfluidic circuit. Figure 1a illustrates a schematic drawing of the CV used in this experiment. The CV is designed with a microsquare of $\sim 50 \mu \mathrm{m} \times \sim 50 \mu \mathrm{m}$, which is used to connect the microchannel from the inlet $A$ by the junction $A$ and the microchannel from the inlet B by the junction B. Both microchannel sidewalls at the junction $\mathrm{A}$ are both $90^{\circ}$ towards the flow at the inlet $\mathrm{A}$, which consequently stops the liquid flow, whereas there is only one sidewall at the junction $B$ that is $90^{\circ}$ towards the flow at the inlet $\mathrm{B}$, which consequently does not stop the liquid flow. With this structure, liquid can flow through the junction B to open the CV and allow the liquid flow in the outlet direction. The different capillary pressure $(\Delta p)$ at the valve junction A can be derived from Eq. (1) as:

$$
\Delta p=2 \gamma \cos \theta_{P M M A}\left(\frac{1}{w_{2}}-\frac{1}{w_{1}}\right),
$$

where $w_{1}$ and $w_{2}$ are the widths of the channels before and after entering the junction, respectively. Here, $w_{1}$ was $\sim 10 \mu \mathrm{m}$, where $w_{2}$ was $\sim 50 \mu \mathrm{m}$. The surface tension of water at $25{ }^{0} \mathrm{C}$ is $72.14 \mathrm{mN} / \mathrm{m}$ (Jasper 1972) and the $\theta_{P M M A}$ with water is $\sim 68^{\circ}$ (Ma et al. 2007) in agreement with PMMA from Goodfellow $\left(\theta_{P M M A, \text { Goodfellow }}=66^{\circ}\right)$ (Soper et al. 2002). By this, giving $\Delta p$ for water was estimated to approximately $-4.3 \mathrm{kPa}$. The principle of the $\mathrm{CV}$ is described in Fig. 1b-g. Considering the microchannel made of a hydrophilic material, the liquid spontaneously flows $\left(\theta_{1}<90^{\circ}\right)$ in the microchannel from the inlet A direction (see Fig. 1b), driven by the positive capillary pressure as previously mentioned above. Then, the liquid meniscus continuously fills the channel and reaches the junction A, where it stops (Fig. 1c) because the contact angle of the flow changes to $\theta_{2}$ $>90^{\circ}$ (Irimia 2008) resulting in a negative capillary pressure (Leu and Chang 2004). Consequently, this CV is closed. Figure 1d illustrates the liquid filling the microchannel before entering the junction B. At this junction (Fig. 1e), the contact angle $\theta_{1}$ at one sidewall changes to $\theta_{3}>90^{\circ}$ resulting in the negative capillary 
pressure, which slows down the liquid flow. However, this negative capillary pressure is not enough to stop the flow due to the positive capillary pressure $\left(\theta_{1}<\right.$ $90^{\circ}$ ) from the wetting liquid at the sidewall in the same direction. Once the liquid wets the sidewall at the junction $B$, the liquid meniscus fills the microsquare of the $\mathrm{CV}$, as depicted in Fig. 1f. When this liquid meniscus touches the liquid meniscus at the junction $\mathrm{A}$, the liquid flows in the outlet direction (the $\mathrm{CV}$ is opened). Figure $1 \mathrm{~h}$ illustrates an optical micrograph of red-dyed water meniscus stopped at the junction $\mathrm{A}$, after dropping on the inlet reservoir connected to the inlet $\mathrm{A}$. As shown in Fig. 1i, the red-dyed water flowed to the outlet when it was dropped on the inlet reservoir connected to the inlet $\mathrm{B}$.

\section{Results and discussion}

A microfluidic circuit has been developed to conceptionally test an automatic labon-chip operation (ALOCO), which enables a user to consecutively load different solutions into the analysis volumes contained in a field of view (FOV). This chip integrated the passive components such as inlet reservoirs, analysis chambers, capillary pump and valves, as well as outlet reservoirs, into a single chip. Figure 2a illustrates a simplified schematic diagram of the ALOCO design. This pattern had three inlet reservoirs (IRA, IRB and IRC) of $\sim 500 \mu \mathrm{m} \times \sim 500 \mu \mathrm{m}$ for successive dropping of three different liquids e.g. assay sample to IRA, washing solution to IRB, and reagent to IRC. In the FOV, 16 analysis chambers (AC1-AC16) of $\sim 50$ $\mu \mathrm{m} \times \sim 50 \mu \mathrm{m}$, where 16 different analytes could be deposited, were connected together in series. This allows the ALOCO device to perform 16 tests from a single serum sample in parallel. The capillary valve system, which consisted of CV1-CV5 as presented in Fig. 2a, controlled the flow of the liquids to the analysis chambers in the FOV. The junction $\mathrm{J} 1$ of $\sim 50 \mu \mathrm{m} \times \sim 50 \mu \mathrm{m}$ was used to combine three separate streams from the inlet reservoirs $\mathrm{A}-\mathrm{C}$ and fed them to the $\mathrm{AC} 1-\mathrm{AC} 16$ in the FOV. The other junctions were used to change the width of the channel from $\sim 20 \mu \mathrm{m}$ to $\sim 10 \mu \mathrm{m}$ (J2 and J3) and from $\sim 10 \mu \mathrm{m}$ to $\sim 20 \mu \mathrm{m}$ (J4). After flow through the FOV, the liquids continuously filled the capillary pump as a waste. The air outlets were used to drain the air (inside the channels) to the atmospheric environment as the channels were filled with the liquids.

To examine the ALOCO design, an open channel microstructure without a PMMA cap was produced as depicted in Fig. 2b. In principle, the capillary pressure only requires a positive value in order to propel liquids through the channels (Sainiemi et al. 2008). To avoid the negative capillary pressure, it was necessary to enclose the open microchannels since the contact angle of the wall with air is $180^{\circ}$. Figure 2c demonstrates a part of the ALOCO device, as marked in Fig. 2b, after bonding with a 50- $\mu \mathrm{m}$-thick PMMA film. This closed channel device was produced in PMMA and $\mathrm{Si}$, which are hydrophilic, $\theta_{P M M A}$ for water $=\sim 68^{\circ}$ (Ma et al. 2007), $\theta_{P M} M A$,Goodfellow (PMMA from Goodfellow) for water $=66^{\circ}$ (Soper et al. 2002) and $\theta_{S i}$ for water $=77.3^{\circ}$ (Cao et al. 2008). This chip ensured spontaneous flow of liquid through the microchannels under the action of capillary forces (Puttaraksa et al. 2013). Figure 2b is a scaled ALOCO chip and Fig. 2c highlights the integrity of the chip after bonding.

Before investigating the flow of liquids in the whole ALOCO chip, a test chip was fabricated (Fig. 3 and 4) for examining the capillary flow through a series of 
16 analysis chambers in the FOV and capillary pump (CP). This device comprised an inlet reservoir connected to the FOV and the CP but no capillary valve system. The CP can be based on a microfluidic chamber filled with a number of structures such as posts, tree lines and hexagons, as reported by Zimmermann et al. (2007). Here, the tree line structure consisting of multiple equivalent microchannels was selected for the CP because of rapid fabrication using the PPAL method. This CP still maintains its function, even if individual channels have single blockages. The test chip performance was characterized by dropping un-dyed DI water onto the inlet reservoir and following the flow under video microscopy. The result of this experiment demonstrated the liquid flow through three microsquare types with different outlet structures, which were used for the analysis chambers in the FOV. The basic design of the three microsquare types was based on the microsquare used in the capillary valve (CV) without the microchannel from the inlet A (see Fig. 1a). The microsquare type I was used in the analysis chambers of AC1, AC2, AC3, AC6, AC7, AC10, AC11, AC14 and AC15, which consisted of a $\sim 50 \mu \mathrm{m} \times \sim 50 \mu \mathrm{m}$ microsquare connected with the inlet channel and the outlet channel in the same flow direction of the inlet. The microsquare type II (AC4, AC5, AC12 and AC13) composed of a $\sim 50 \mu \mathrm{m} \times \sim 50 \mu \mathrm{m}$ microsquare connected with the inlet channel and the outlet channel in $90^{\circ}$ flow direction from the inlet, whereas the microsquare type III was applied in the analysis chambers of AC8, AC9 and AC16. The outlet flow direction of the microsquare type II was similar as the microsquare type III, which changed the flow direction in $90^{\circ}$ from the inlet. However, the outlet channel of the microsquare type II was connected to the corner of the microsquare opposite to the corner of the inlet channel, whereas the outlet channel of the microsquare type III was connected to the corner of the microsquare next to the corner of the inlet channel (see detail in Fig. 2a). Figure 3a illustrates when the un-dyed water was flowing through the first analysis chamber AC1 (the microsquare type I). Then, the un-dyed water meniscus continuously filled the $\sim 10-\mu \mathrm{m}-$ wide and $\sim 200 \mu \mathrm{m}-$ long-channel, which was used to connect the first analysis chamber (AC1) and the second analysis chamber (AC2). As shown in Fig. 3b, the liquid meniscus started to fill the AC2 (the microsquare type I). This liquid meniscus filling the junction of the $\mathrm{AC} 2$ was similar to the liquid filling the junction B of the $\mathrm{CV}$, as depicted in Fig. 1e. Subsequently, the liquid meniscus filled the microsquare of the AC2 (see Fig. $3 \mathrm{c}$ ), which was agreed to the liquid meniscus filling the microsquare to open the CV (see Fig. 1f). Then, the un-dyed water flowed through the AC5 (the microsquared type II), as illustrated in Fig. 3d. Figure 3e presents the liquid meniscus filling the AC9 (the microsquared type III). After filling all the 16 analysis chambers, the un-dyed water flowed into the straight channel with $\sim 20 \mu \mathrm{m}$ width before flowing to the CP, see Fig. 3f. For this straight channel, the volumetric flow rate was evaluated to $10 \pm 2 \mathrm{~nL} / \mathrm{min}$. This is lower than the flow rate of the microfluidic chip made by PDMS surface oxidation $\left(\theta_{\text {oxidized-PDMS }}=40^{\circ} \pm 5^{\circ}\right)$, reported by Novo et al. (2013). This is consistent with the greater contact angle of PMMA and $\mathrm{Si}$, although different channel geometry may also influence the flow rate. It is demonstrated in Fig. 3a-f that a series of microsquares was correctly filled with undyed water without generation of bubbles, which would influence biological assays. The CP was progressively filled channel by channel with the un-dyed water as demonstrated in Fig. $4 \mathrm{a}-\mathrm{c}$. It is seen that no trapping of air in the main channels of the CP was observed. This implies that the pumped volume was precisely defined lithographically. This is more precise than using a porous fiber pad where the 
volume of liquid absorption is governed by ill-defined manufacturing tolerances. The CP volume should accommodate the total volume of assay sample, washing solution, and reagent that have flown through the microfluidic circuit. For 1:1 channel width:channel wall thickness with 10- $\mu$ m-deep channels, this corresponds to about $2 \mathrm{~cm}^{2} / \mu \mathrm{L}$. Hence, even large total volume $(15 \mu \mathrm{L})$ can be handled on a chip with the same size as a standard credit card. Here, the test ALOCO circuit was fabricated with a small CP because the size of the chip was limited by the limits of travel for the PPAL system linear positioners (Gorelick et al. 2008).

The main function of the ALOCO chip is to manipulate the liquids, which were successively dropped in different three inlet reservoirs (IRA, IRB, and IRC), to flow through the FOV. This principle is controlled by the capillary valves (CV1CV5). Previously, passive valves, e.g. capillary valves (Zimmermann et al. 2008), hydrophobic valves (Feng et al. 2003) and air bubble valves (Xu et al. 2012), were applied in microfluidics. Instead of using these valves, air-trapped bubbles were used to separate and control the sequential flows of three liquid solutions to a detection chamber (Novo el al. 2013). The method of using the air-trapped bubbles is simple. However, air bubbles could block and influence the flow in microfluidic channels (Chang et al. 2008; Khoshmanesh et al. 2015). Additionally, an avoidance of air bubbles is necessarily required in some applications, e.g. cell culture (Sung and Shuler 2009) and polymerase chain reaction (Liu et al. 2007). By this, the capillary valve is more advantageous. According to our best knowledge, this ALOCO chip was the first design that employed the capillary passive valves for manipulating different liquid streams, which were fed to a detection area. The ALOCO design is beneficial e.g. in an immunoassay, where the contamination of washing solutions can be reduced in the washing steps. Moreover, the left over from the previous flow steps as well as the unspecific bindings of the biological samples and reagents can also be decreased using the ALOCO device. To test this chip and manipulation of different liquids, the ALOCO device was tested with red-, blue- and un-dyed DI water. At first, the red-dyed water was dropped on the IRA. By the capillary forces, the red-dyed water filled the $\sim 20-\mu$ m-wide channel before flowing to the CV1 (see detail in Fig. 2a). Then, the CV1 was opened, which allowed the red-dyed water filling of J1. After flow through the J1, the red-dyed water meniscuses stopped at the CV2 and CV3, resulting in the flow towards the FOV. As illustrated in Fig. 5a, the fifteenth analysis chamber (AC15) was filled with the red-dyed water. Subsequently, the blue-dyed water was dropped on the IRB. After that, the blue-dyed water flowed through the J2 and continuously filled the channel to open the CV2. When the CV2 was open, the blue-dyed water meniscus progressively flowed and stopped at the CV5. By this, the blue-dyed water moved to the FOV, as depicted in Fig. 5b. The flow of blue-dyed water for opening the CV2 and stopping at the CV5 agreed well with the CV principle as previously described (see also Fig. 1b-g). After the blue-dyed water flow through the sixteenth analysis chamber (AC16), un-dyed water was consequently dropped on the IRC. Figure 5c reveals that the blue-dyed water completely replaced the red-dyed water in the FOV and the channel in the direction of the IRA. The flow of blue-dyed water into the channel of IRA direction could suggest that the reddyed water flow in the outlet direction in Fig. 1i was from the flow of red-dyed water in the inlet $\mathrm{B}$ direction. As demonstrated also in the right bottom of Fig. $5 \mathrm{c}$, the un-dyed water filled the $\sim 20-\mu \mathrm{m}$-wide channel. Then, it filled the J3 and opened the CV3. After that, the un-dyed water flowed towards the J1 and stopped 
at the CV4. Unfortunately, the CV4 opened after a period of $41.13 \mathrm{~s}$, resulting in the un-dyed water flowing towards the air outlet as depicted in Fig. 5d. The cause of this is unclear, but it might be associated with sudden increase in flow resistance rupturing the meniscus in the CV4 due to saturation of the CP. This effect may be mitigated by using a larger capacity of the CP and/or increasing the width $w_{2}$ (see Fig. 1a) for CV4 and CV5.

\section{Conclusions}

We have demonstrated a new microfluidic design of an automatic lab-on-chip operation (ALOCO) device based on manipulating liquids using capillary forces. The chip was produced by programmable proximity aperture lithography (PPAL) technique and its operation tested with red-, blue- and un-dyed DI water. The ALOCO design is the first time that uses the capillary valve system to control the directions of liquids flowing from different streams to a common analysis area. The ALOCO device enables 16 simultaneous tests from one analyzed sample by passive operation. Novelties in the specific design features, such as valve, capillary pump, air outlets, consecutive analysis chambers, allow an easy-to-use automatic lab-on-chip device that has diverse potential as a passive diagnostic point-of-care assay, such as seen in the seventh framework programme of the European Union (FP7/EU) hilysens project.

Acknowledgements This work was partially funded by European union (EU) grant agreement number 262411. The funding source had no involvement or role in the development of this article. Dr. Liping Wang is gratefully acknowledged for conceptualization of the microfluidic design.

\section{References}

Abramoff MD, Magelhaes PJ, Ram SJ (2004) Image processing with ImageJ. Biophoton Int $11: 36-42$

Ansari K, van Kan JA, Bettiol AA, Watt F (2006) Stamps for nanoimprint lithography fabricated by proton beam writing and nickel electroplating. Journal of Micromechanics and Microengineering 16:1967-1974

Becker H, Heim U (2000) Hot embossing as a method for the fabrication of polymer high aspect ratio structures. Sensors and Actuators 83: 130-135

Bousse L, Cohen C, Nikiforov T, Chow A, Kopf-Sill AR, Dubrow R, Wallace Parce J (2000) ELECTROKINETICALLY CONTROLLED MICROFLUIDIC ANALYSIS SYSTEMS. Annu Rev Biophys Biomol Struct 29:155-181

Brun S, Savu V, Schintke S, Guibert E, Keppner H, Brugger J, Whitlow HJ (2013) Application of stencil masks for ion beam lithographic patterning. Nucl Instr Meth B 306:292-295

Cao L, Price TP, Weiss M, Gao D (2008) Super Water- and Oil-Repellent Surfaces on Intrinsically Hydrophilic and Oleophilic Porous Silicon Films. Langmuir 24:1640-1643

Chang FM, Sheng YJ, Cheng SL, Tsao HK (2008) Tiny bubble removal by gas flow through porous superhydrophobic surfaces: Ostwald ripening. Appl Phys Lett 92:264102

Chin CD, Linder V, Sia SK (2007) Lab-on-a-chip devices for global health: Past studies and future opportunities. Lab Chip 7:41-57

Cho YK, Lee JG, Park JM, Lee BS, Lee Y, Ko C (2007) One-step pathogen specific DNA extraction from whole blood on a centrifugal microfluidic device. Lab Chip 7:565-573

Delamarche E, Bernard A, Schmid H, Bietsch A, Michel B, Biebuyck H (1998) Microfluidic Networks for Chemical Patterning of Substrates: Design and Application to Bioassays. J 
Am Chem Soc 120:500-508

Dittrich PS, Manz A (2006) Lab-on-a-chip: microfluidics in drug discovery. Nature Reviews Drug Discovery 5:210-218

Feng Y, Zhou Z, Ye X, Xiong J (2003) Passive valves based on hydrophobic microfluidics. Sensors and Actuators A 2003:138-143

Gorelick S, Puttaraksa N, Sajavaara T, Laitinen M, Singkarat S, Whitlow HJ (2008) Fabrication of microfluidic devices using $\mathrm{MeV}$ ion beam Programmable Proximity Aperture Lithography (PPAL). Nucl Instr Meth B 266:2461-2465

Irimia D (2008) Capillary Force Valves. Encyclopedia of Microfluidic and Nanofluidics 192-196. Jasper JJ (1972) The Surface Tension of Pure Liquid Compounds. Journal of physical and chemical reference data 1(4):841-1009

Jiang H, Weng X, Li D (2011) Microfluidic whole-blood immunoassays. Microfluid Nanofluid 10:941-964

Jin S, Dai M, He Fei, Wang Y, Ye BC, Nugen SR (2012) Development and characterization of a capillary-flow microfluidic device for nucleic acid detection. Microsyst Technol 18:731-737 Juncker D, Schmid H, Drechsler U, Wolf H, Wolf M, Michel B, Rooij ND, Delamarche E (2002) Autonomous Microfluidic Capillary System. Anal Chem 74:6139-6144

Khoshmanesh K, Almansouri A, Albloushi H, Yi P, Soffe R, Kalantar-Zadeh K (2015) A multi-functional bubble-based microfluidic system. SCIENTIFIC REPORTS 5 (9942):1-8 Leu TS, Chang PY (2004) Pressure barrier of capillary stop valves in micro sample separators. Sensors and Actuators A 115: 508-515

Liu HB, Gong HQ, Ramalingam N, Jiang Y, Dai CC, Hui Km (2007) Micro air bubble formation and its control during polymerase chain reaction (PCR) in polydimethylsiloxane (PDMS) microreactors. Journal of Micromechanics and Microengineering 17:2055

Ma Y, Cao X, Feng X, Ma Y, Zou H (2007) Fabrication of super-hydrophobic film from PMMA with intrinsic water contact angle below $90^{\circ}$. Polymer 48:7455-7460

Mouradian S (2001) Lab-on-a-chip: applications in proteomics. Current Opinion in Chemical Biology 6:51-56

Novo P, Volpetti F, Chu V, Conde JP (2013) Control of sequential fluid delivery in a fully autonomous capillary microfluidic device. Lab Chip 13:641-645

Puttaraksa N, Gorelick S, Sajavaara T, Laitinen M, Singkarat S, Whitlow HJ (2008) Programmable proximity aperture lithography with MeV ion beams. J Vac Sci Technol B 26(5):1732-1739

Puttaraksa N, Unai S, Rhodes MW, Singkarat K, Whitlow HJ, Singkarat S (2012) Fabrication of a negative PMMA master mold for soft-lithography by $\mathrm{MeV}$ ion beam lithography. Nucl Instr Meth B 272:149-152

Puttaraksa N, Napari M, Meriläinen L, Whitlow HJ, Sajavaara T, Gilbert L (2013) High speed microfluidic prototyping by programmable proximity aperture $\mathrm{MeV}$ ion beam lithography. Nucl Instr Meth B 306:302-306

Sackmann EK, Fulton AL, Beebe DJ (2014) The present and future role of microfluidics in biomedical research. Nature 507:181-189

Sainiemi L, Nissilä T, Jokinen V, Sikanen T, Kotiaho T, Kostiainen R, Ketola RA, Franssila S (2008) Fabrication and fluidic characterization of silicon micropillar array electrospray ionization chip. Sensors and Actuators B 132:380-387

Squires TM, Quake SR (2005) Microfluidics: Fluid physics at the nanoliter scale. Rev Mod Phys 77(3):977-1026

Soper SA, Henry AC, Vaidya B, Galloway M, Wabuyele M, McCarley RL (2002) Surface modification of polymer-based microfluidic devices. Analytica Chimica Acta 470:87-99

Srinivasan V, Pamula VK, Fair RB (2004) An integrated digital microfluidic lab-on-a-chip for clinical diagnostics on human physiological fluids. Lab Chip 4:310-315

Stone HA, Stroock AD, Ajdari A (2004) ENGINEERING FLOWS IN SMALL DEVICES: Microfluidics Toward a Lab-on-a-Chip. Annu Rev Fluid Mech 36:381-411

Sung JH, Shuler ML (2009) Prevention of air bubble formation in a microfluidic perfusion cell culture system using a microscale bubble trap. Biomed Microdevices 11:731-738

Weigl BH, Bardell RL, Cabrera CR (2003) Lab-on-a-chip for drug development. Advanced Drug Delivery Reviews 55:349-377

Whitesides GM (2006) The origins and the future of microfluidics. Nature 442:368-373

Whitlow HJ, Wang LP, Gilbert L (2009) Transport of water and particles in microfluidics devices lithographically fabricated using proton beam writing (PBW). Advanced Materials Research 74: 129-132 
Xu Y, Lv Y, Wang L, Xing W, Cheng J (2012) A microfluidic device with passive air-bubble valves for real-time measurement of dose-dependent drug cytotoxicity through impedance sensing. Biosensors and Bioelectronics 32:300-304

Yeo LY, Friend JR (2009) Ultrafast microfluidics using surface acoustic waves. Biomicrofluidics 3:012002-1-012002-23

Zimmermann M, Schmid H, Hunziker P, Delamarche E (2007) Capillary pumps for autonomous capillary systems. Lab Chip 7:119-125

Zimmermann M, Hunziker P, Delamarche E (2008) Valves for autonomous capillary systems. Microfluid Nanofluid 5:395-402

Zimmermann M, Hunziker P, Delamarche E (2009) Autonomous capillary system for one-step immunoassays. Biomed Microdevices 11:1-8 
(a)

\section{Inlet B}

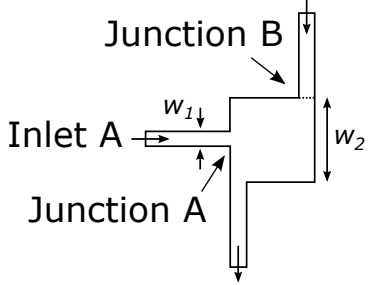

(d)

Outlet

\section{Second liquid flow}

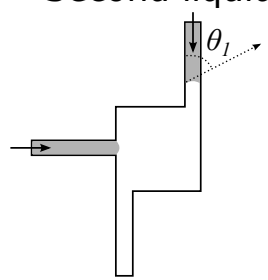

(b)

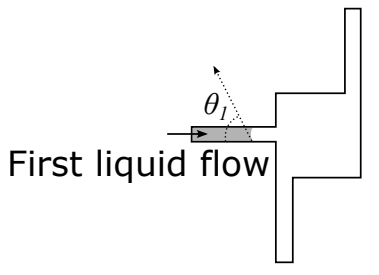

(e)

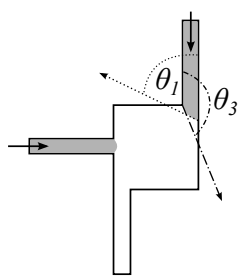

(c)

Liquid meniscus stopped at the junction $\mathrm{A}$

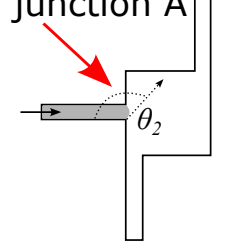

(f)

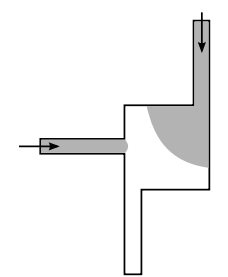

(g)

\section{Second liquid flow}

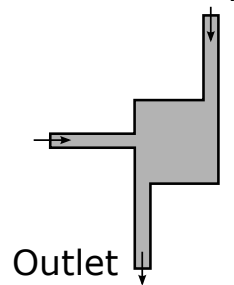

(h)

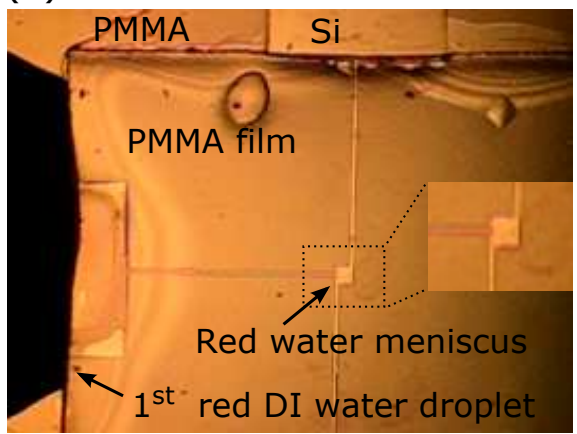

(i)

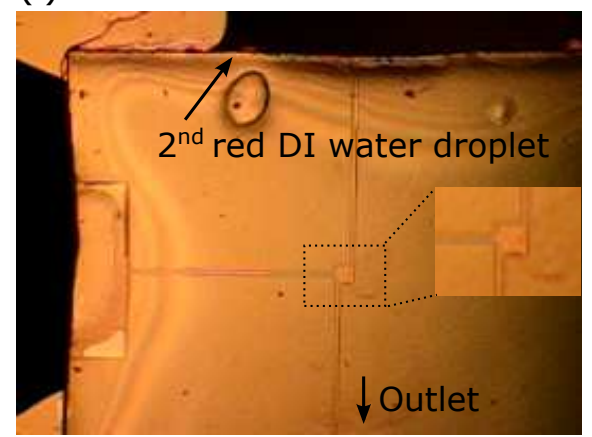

Fig. 1 Capillary valve (CV) designed for the ALOCO devices. (A) Schematic illustration of the CV consisting of $10-\mu \mathrm{m}$-wide channels $\left(w_{1}\right)$ of two inlets and one outlet, which are connected to a microsquare of $\sim 50 \mu \mathrm{m} \times \sim 50 \mu \mathrm{m}\left(w_{2}\right)$. Sketches of the CV operation principle: (B) the first liquid flow in the inlet A direction, (C) the first liquid flow stop at the junction $\mathrm{A}$ as indicated by the red arrow, (D) the second liquid flow in the inlet B direction, (E) the second liquid meniscus filling the junction $\mathrm{B},(\mathrm{F})$ the liquid meniscus filling a microsqure to open the $\mathrm{CV}$ and $(\mathrm{G})$ the liquid flow in the outlet direction after touching between two liquid menisci from the inlet $\mathrm{A}$ and $\mathrm{B} . \theta_{1}<90^{\circ}$ is the contact angle of the liquid in a hydrophilic microchannel. $\theta_{2}>90^{\circ}$ is the changed contact angle at the sidewalls in junction A. $\theta_{3}>90^{\circ}$ is the changed contact angle at one sidewall at junction $\mathrm{B}$. (H) The valve was closed when the first liquid filled in the direction of inlet $\mathrm{A}$. The panel provides microscopic image of the retarded red water meniscus at the valve junction with the magnified image in the marked area. (I) The valve was open when the second liquid filled in the direction of inlet B. The panel demonstrates that the valve opened and the red water passed to the outlet. The right image inside the panel illustrated a magnified region of the valve 
(a) Air outlet Air outlet
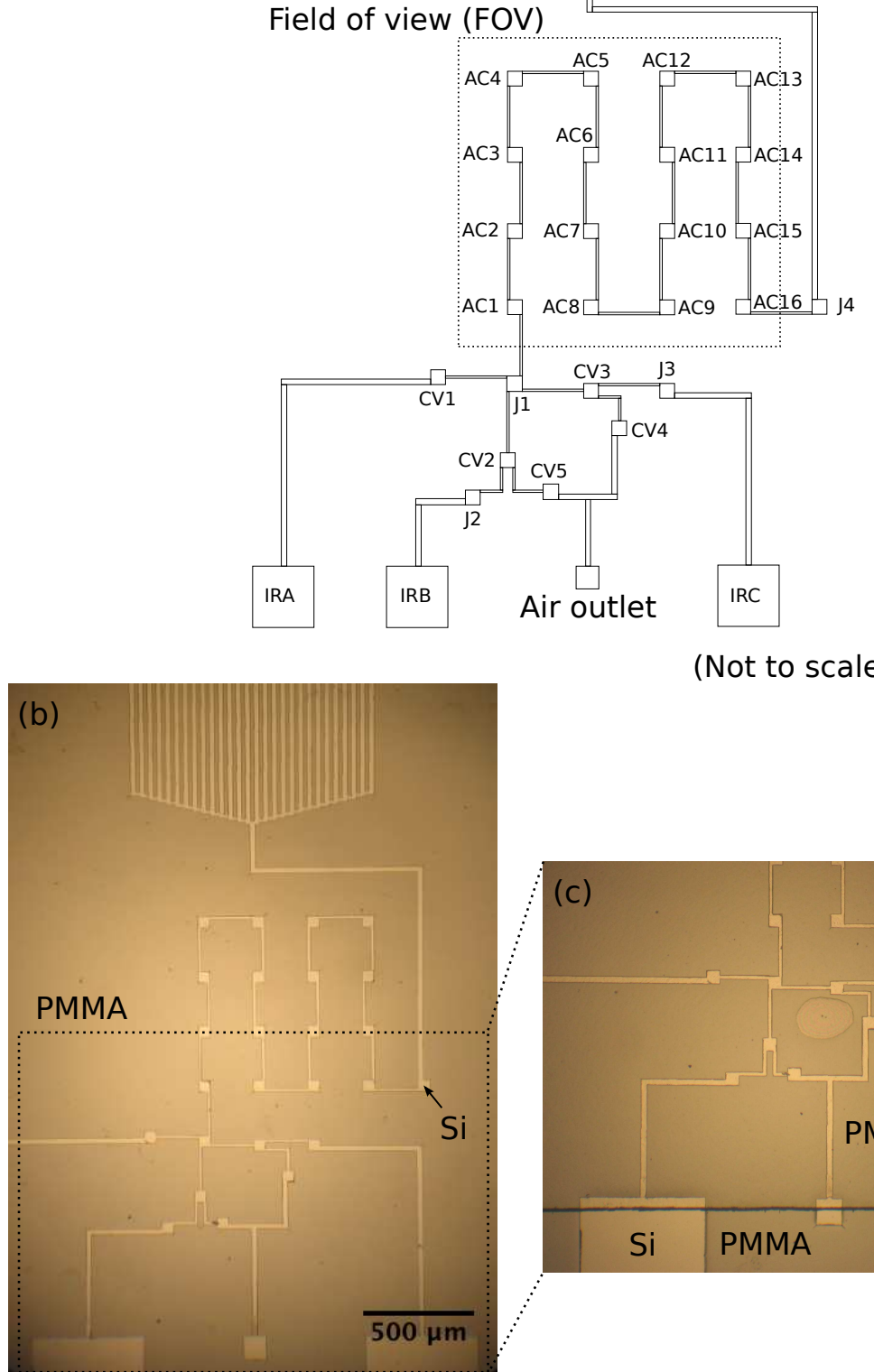

(Not to scale)

Fig. 2 (A) An automatic lab-on-chip operation (ALOCO) design with integrating the passive microfluidic components. Three inlet reservoirs (IRA, IRB and IRC), the junctions (J1-J4), the capillary valves (CV1-CV5), the analysis chambers (AC1-AC16), the capillary pump, and three air outlet reservoirs were illustrated. (B) Experimental optical micrograph of the ALOCO device fabricated using the PPAL method. Two reservoirs, an air outlet, junctions, capillary valves, 16 analysis chambers, and the capillary pump, as ascribed in Fig. (A), were displayed. (C) A magnified image of the region illustrated dotted in Fig. (B) after bonding with a $50-\mu \mathrm{m}-$ thick PMMA capping film 

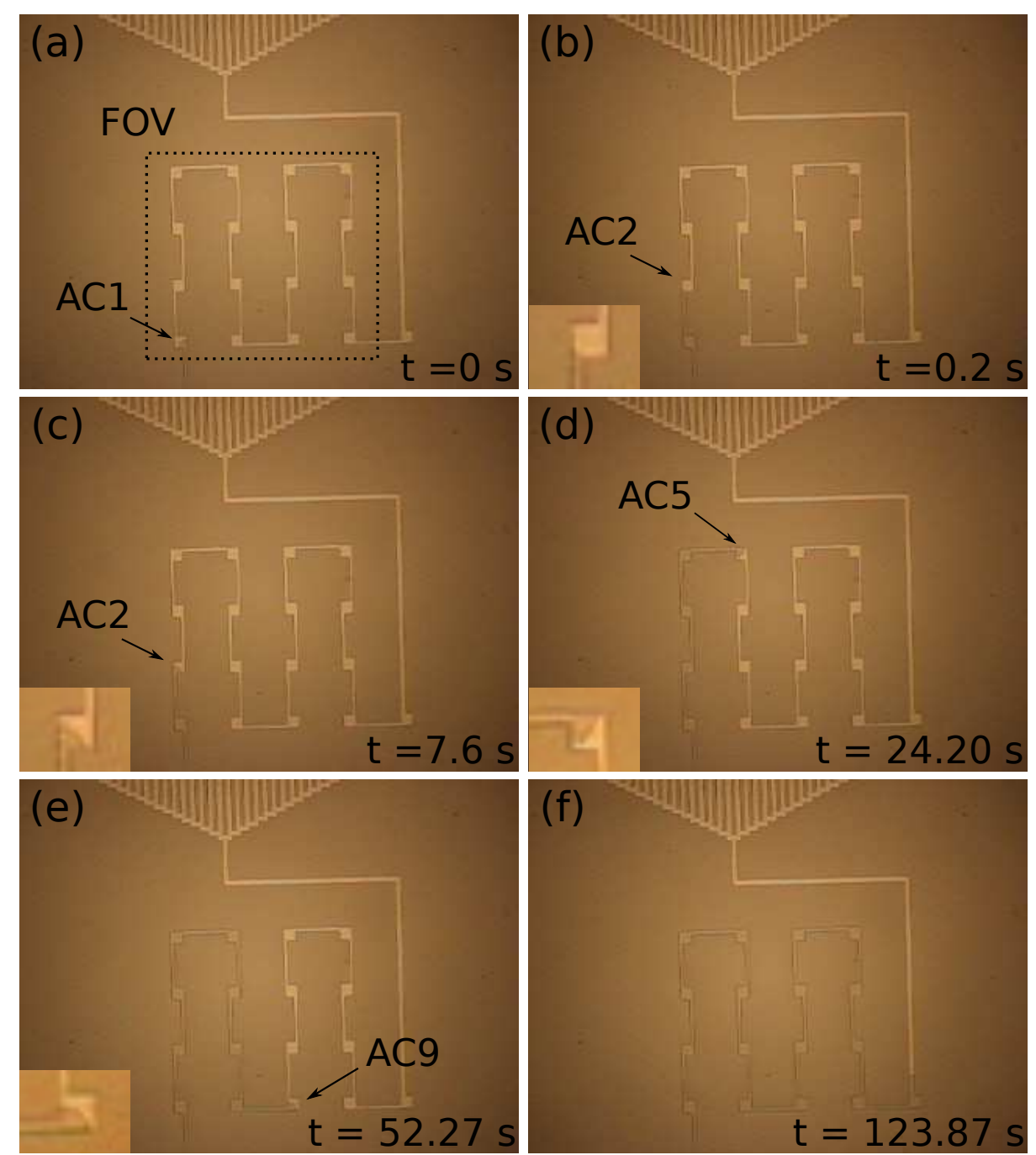

Fig. 3 Passive real-time images of the analysis chambers during filling with un-dyed DI water. Un-dyed water filling: (A) the analysis chamber $\mathrm{AC} 1$ (the microsquare type I), (B) the junction of $\mathrm{AC} 2,(\mathrm{C})$ the analysis chamber $\mathrm{AC} 2$ (the microsquare type $\mathrm{I}$ ), (D) the analysis chamber AC5 (the microsquare type II), (E) the analysis chamber AC9 (the microsquare type III) and (F) the $\sim 20-\mu$ m-wide channel towards extending from the FOV to the capillary pump. The bottom-left images inside the panels (B), (C), (D), and (E) are magnified images of the un-dyed water filled the junction $\mathrm{AC} 2$, the microsquare type $\mathrm{I}(\mathrm{AC} 2)$, the microsquare type II (AC5), and the microsquare type III (AC9), respectively 

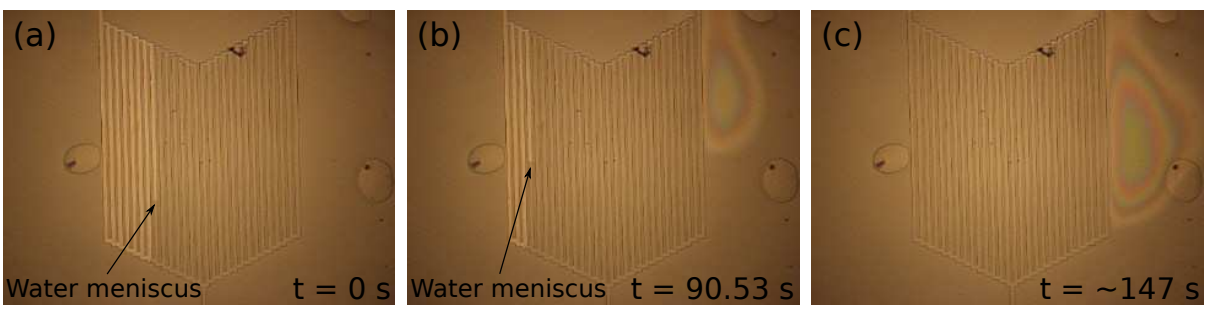

Fig. 4 Passive filling the capillary pump. Optical images when the un-dyed DI water meniscus was filling the channels of the capillary pump at $(\mathrm{A}) \mathrm{t}=0 \mathrm{~s},(\mathrm{~B}) \mathrm{t}=90.53 \mathrm{~s}$, and $(\mathrm{C}) \mathrm{t}=$ $\sim 147$ s, respectively
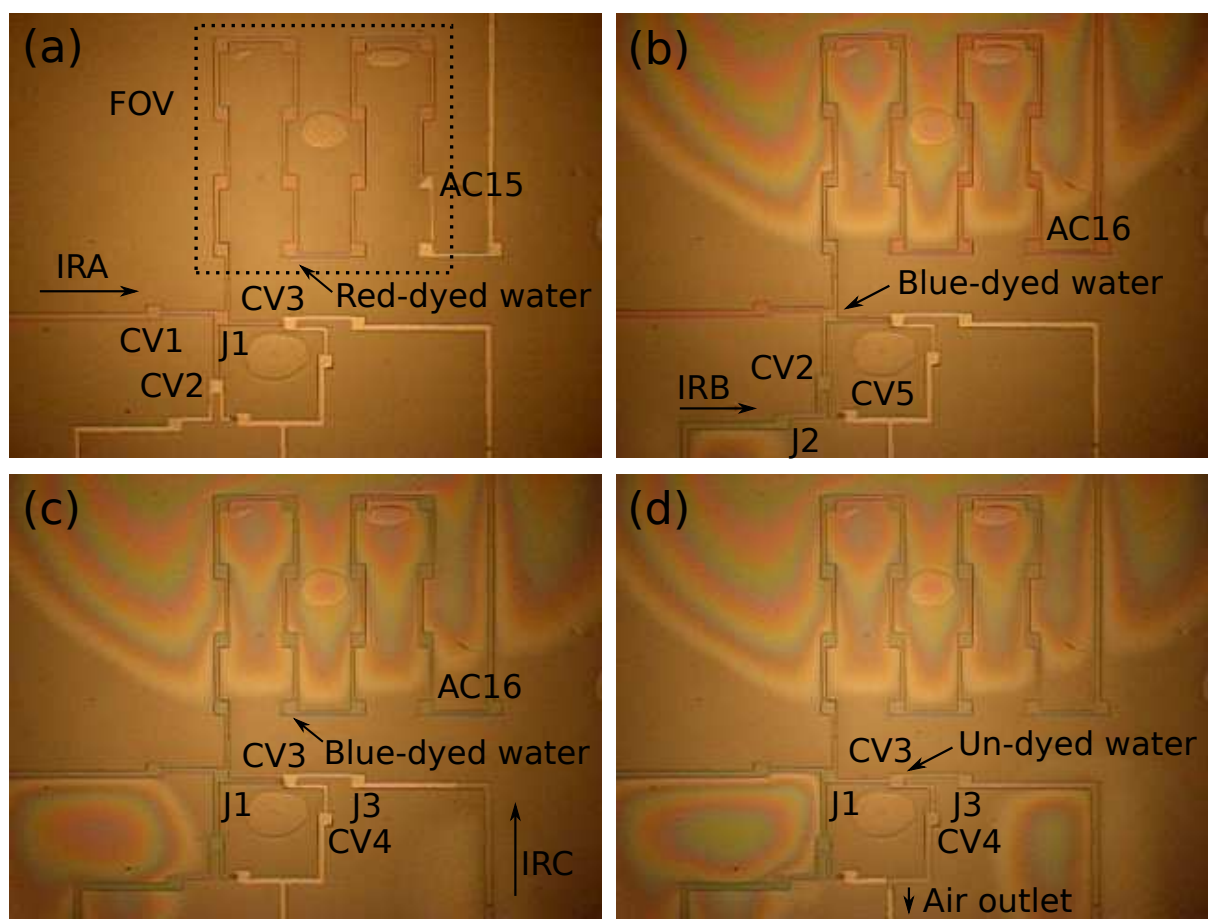

Fig. 5 Directional control of liquids in the ALOCO device. (A) Red-dyed DI water flowed towards the FOV, after dropping onto the inlet reservoir A (IRA), see Fig. 2a. (B) Blue-dyed DI water filled the microchannels towards the FOV, after dropping onto the inlet reservoir B (IRB). (C) Un-dyed DI water filled the microchannel, after dropping over the inlet reservoir $\mathrm{C}$ (IRC). The blue-dyed water flow from the IRB completely substituted the red-dyed water in the FOV. (D) Un-dyed water filled the microchannel towards the J1. Accidentally, the CV4 was open, allowing the un-dyed water filling the microchannel towards the air outlet 